\title{
Left Atrial-Esophageal Fistula Repair after Radiofrequency Catheter Ablation for Atrial Fibrillation
}

\author{
Jeffrey B. Velotta ${ }^{1}$ Charles R. Vasquez ${ }^{2}$ Ralph M. Bolman ${ }^{3}$ Marcelo C. Dasilva ${ }^{1}$
}

${ }^{1}$ Department of Thoracic Surgery, Brigham and Women's Hospital, Boston, Massachusetts, United States

2 Dana Farber Cancer Institute, Boston, Massachusetts, United States

${ }^{3}$ Department of Cardiac Surgery, Brigham and Women's Hospital, Boston, Massachusetts, United States

Address for correspondence Jeffrey B. Velotta, MD, Department of Thoracic Surgery, Brigham and Women's Hospital, 75 Francis Street, Boston, MA 02115, United States (e-mail: jvelotta@partners.org).

Thorac Cardiovasc Surg Rep 2013;2:13-15.
Abstract
Keywords
- ablative therapy
- atrial fibrillation
- atrial-esophageal
- esophageal surgery
- thoracotomy

Left atrial-esophageal fistula (LAEF) is a rare complication of radiofrequency ablation (RFA) procedures undertaken for atrial fibrillation (AF). This complication is associated with significant morbidity and mortality. Currently, there is no clear consensus on the appropriate management strategy. We report a case of a LAEF that developed in a patient 2 weeks after RFA for medication refractory AF. The patient underwent successful repair of the fistula through a left posterolateral thoracotomy, wherein the esophageal and atrial lesions were repaired primarily with an intercostal muscle flap and bovine pericardial patch to reinforce and prevent recurrence.

\section{Introduction}

Left atrial-esophageal fistula (LAEF) after radiofrequency ablation (RFA) is a rare complication associated with high mortality if not recognized early and managed with definitive surgical repair. We present a case of LAEF successfully managed with urgent surgical repair and will outline the clinical course to avoid future pitfalls.

\section{Case Report}

A 72-year-old woman with a history of paroxysmal atrial fibrillation (AF) was referred to this institution after developing frequent symptomatic episodes of AF despite treatment with metoprolol and flecainide. Subsequently, she underwent a successful RFA procedure, with pulmonary vein isolation and isolation of the posterior left atrial wall. In total, the ablation procedure resulted in 188 radiofrequency applications for a total lesion time of 4,845 seconds. There were no immediate postprocedure complications and the patient was discharged home on metoprolol, warfarin (Coumadin), and pantoprazole. Despite the use of pantoprazole, the patient began developing worsening odynophagia and heartburn over a period of several days postprocedure. On postprocedure day 8 , the patient developed a fever of $102 \mathrm{~F}$ and presented to an outside hospital emergency department where she underwent computed tomographic (CT) chest imaging with oral and intravenous contrast. This showed a small pocket of extraluminal air adjacent to the subcarinal esophagus with esophageal narrowing and thickening (-Fig. 1). Additional history and laboratory investigation at that time noted a fever of $102 \mathrm{~F}$, an elevated white blood cell count of 14,000 and worsening odynophagia. At this point, she was transferred to our institution for the management of a suspected LAEF. Before transfer, vitamin K was administered due to an elevated international normalized ratio of 2.0.

On arrival at our institution, the patient was noted to be hemodynamically stable, with continued heartburn, odynophagia, pleuritic chest pain, and a fever of $101.5 \mathrm{~F}$. The patient was immediately transferred to the operating room. Percutaneous catheter access was obtained through the left femoral vein for possible cardiopulmonary bypass. The patient was received

May 30, 2013

accepted after revision

September 17, 2013

published online

November 13, 2013
DOI http://dx.doi.org/

10.1055/s-0033-1358779. ISSN 2194-7635. (c) 2013 Georg Thieme Verlag KG
Stuttgart · New York

License terms

((1) $\circledast$ 


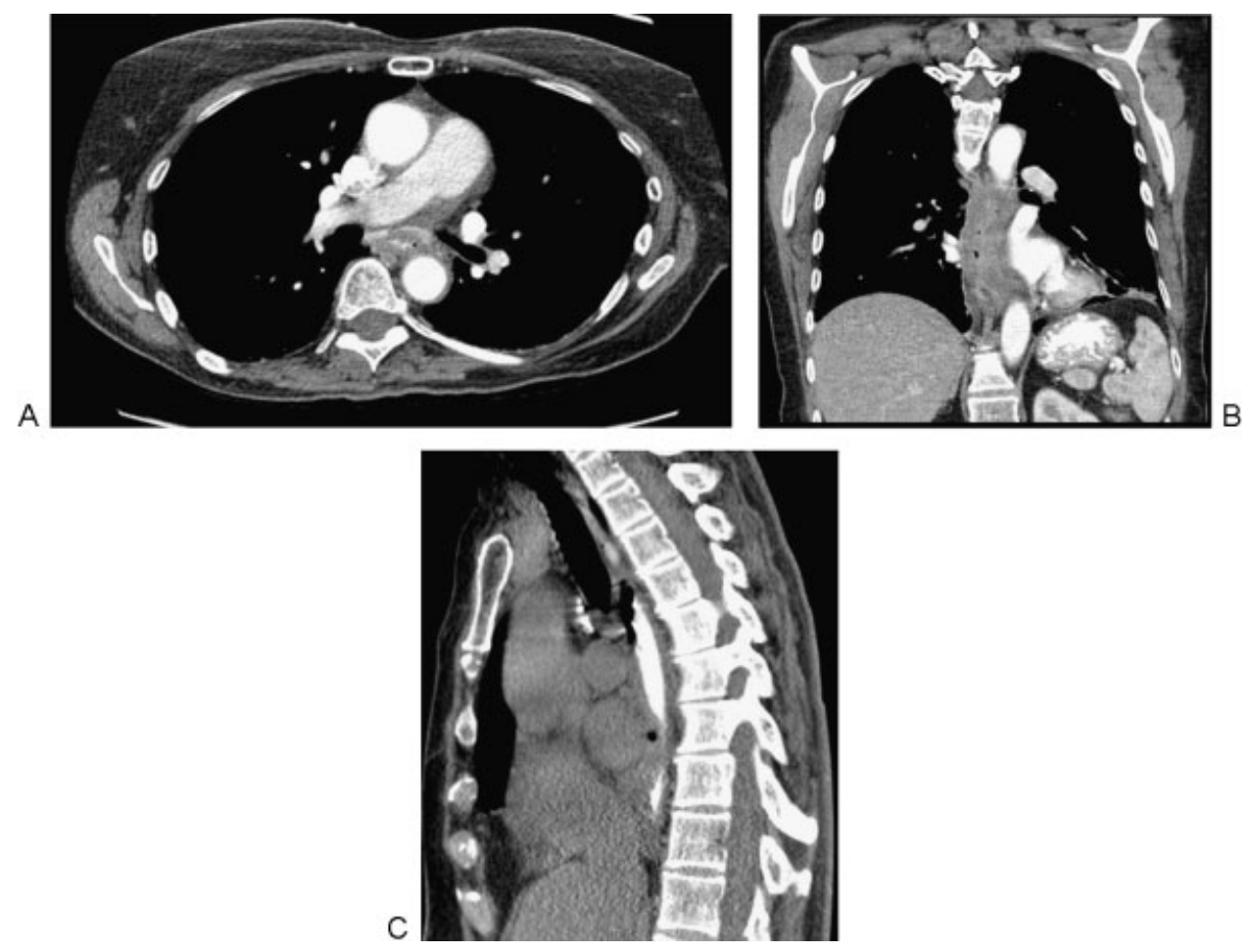

Fig. 1 Computed tomographic image of the chest demonstrates a small pocket of extraluminal air adjacent to the subcarinal esophagus with esophageal narrowing and thickening; (A) axial, (B) coronal, and (C) sagittal.

placed in the right lateral decubitus position and a left posterolateral thoracotomy was performed through the fifth intercostal space. On entry, a pedicled intercostal muscle flap was harvested. An inflammatory effusion of $200 \mathrm{~mL}$ was identified on entering the chest, and a sample was sent for analysis. The inferior pulmonary ligament was dissected and inflamed lymph nodes were identified at levels eight and nine, which were sent for pathologic analysis. After further dissection proximally to the superior pulmonary vein, the esophagus was mobilized, and a small amount of opaque fluid was observed suggestive of mediastinitis. The pericardium was opened longitudinally posterior to the left phrenic nerve, at which time a large amount of purulent fluid was exuded. A sample of this fluid was sent for Gram stain and culture as well. After copious irrigation, two sutures were placed in the pericardium to aid in visualization of the posterior left atrium. A tense 5-mm hematoma was noted on the posterior left atrium, however, not actively bleeding. Directly adjacent to the left atrial lesion was a 4-mm esophageal full thickness injury. Interrogation of the atrial lesion was carefully undertaken and the corresponding injury was repaired using two 3-0 reinforced felt-pledget Prolene (Ethicon Inc., Somerville, New Jersey, United States) sutures. The esophageal lesion was then closed using full thickness interrupted 3-0 PDS (Ethicon Inc.) sutures followed by interrupted 3-0 silk sutures reapproximating the esophageal muscle to complete a two-layer repair. The intercostal muscle flap isolated from the fifth intercostal space was placed over the esophageal repair and secured with a 3-0 Vicryl (Ethicon Inc.). In addition, a $2 \times 9 \mathrm{~cm}$ bovine pericardial patch was placed at the interface between the posterior left atrium and esophageal repair to prevent a fistula recurrence (-Fig. 2). After chest irrigation with $3 \mathrm{~L}$ of normal saline, a 24-French Blake drain was placed adjacent to the esophageal repair, and a chest tube was placed anterior to the apex of the lung to provide additional drainage. The chest and skin incisions were then closed in the standard fashion, and the patient was transferred to the intensive care unit in stable condition.

The patient was extubated on postoperative day (POD) 1 . Triple antibiotic therapy was continued for 7 days. On POD 3, the patient did revert to AF with rapid ventricular rate, which resolved after synchronized cardioversion and administration of amiodarone. Culture results from operating room samples were negative most likely due to previous antibiotic

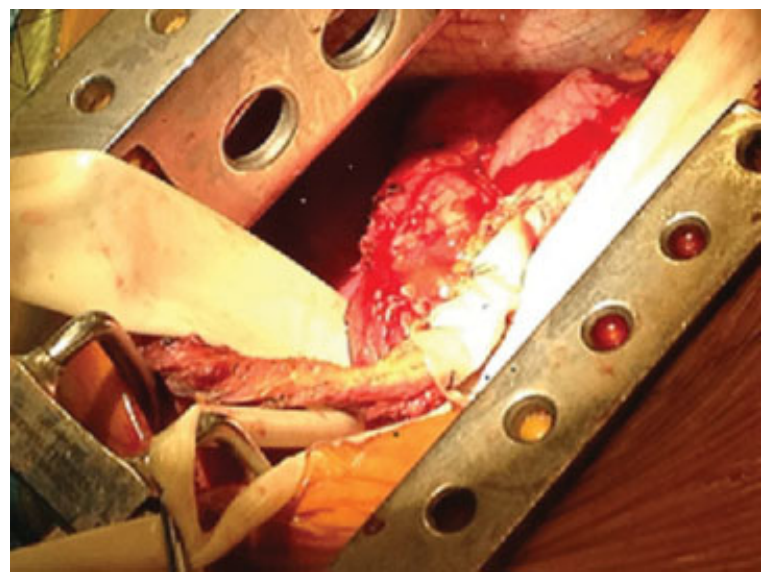

Fig. 2 Completed esophageal repair with intercostal muscle flap and bovine pericardial patch only. 
administration. A thin barium esophagram performed on POD 5 showed no extravasation or obstruction. Clear liquids were started and patient was tolerating a regular diet on POD 6 in which her Blake drain and chest tube were removed. She was discharged home on POD 8 with adequate oral intake and was instructed to continue Coumadin for 6 weeks. At her 6-week follow-up appointment, a postoperative CT scan of the chest with oral contrast demonstrated resolution of the LAEF track and no pleural or pericardial effusions.

\section{Discussion}

Radiofrequency catheter ablation procedures are frequently performed for the treatment of medication refractory AF. Before catheter ablative techniques for the treatment of AF, LAEF was a rare, but devastating complication after open cardiac surgical operations in which RFA devices were used to ablate the re-entrant pathways in the pulmonary veins. ${ }^{1}$ To counteract the extreme thermal energy produced from RFA, various manufacturers and clinicians have switched to cooler temperatures and changes in frequency of energy sources. LAEF after catheter RFA occurs in only 0.3 to $0.4 \%$ of patients undergoing this procedure. ${ }^{2}$ LAEF is associated with high mortality, most frequently due to septic or air emboli to the brain, or less commonly to other organs. ${ }^{3,4}$ Complications also occur as a result of generalized sepsis or massive gastrointestinal bleeding. ${ }^{3,4}$ However, as our case demonstrates, early recognition of this complication, combined with immediate operative repair, can avoid the potential sequelae of LAEF.

Although there is some debate regarding diagnosis of this devastating complication, it is our recommendation to not undergo esophagogastroduodenoscopy (EGD) or transesophageal echocardiography (TEE) before repair given the high likelihood of inducing a massive air embolus. Barium esophagram or CT chest with oral contrast should be considered as first-line imaging modalities to detect LAEF. In terms of treatment options, there have been both nonsurgical and surgical treatment methods for patients with LAEF. Most of the literature discuss surgical repair of LAEF after catheter ablation. However, a recent case series reported the successful use of endoscopically placed esophageal stents and pericardial drains in patients with esophagopericardial fistulas. ${ }^{5}$

A survey of the literature reveals multiple surgical approaches to repair of LAEF. We chose to approach the lesion through a left thoracotomy due to the presence of a concurrent left pleural effusion, but a right thoracotomy may provide similar access. Given the potential for massive bleeding from an atrial perforation, we chose to complete the operative repair with cardiopulmonary bypass on standby. As was performed in this case, we advocate that a biologic barrier be placed between the esophageal and atrial repair to prevent refistulization. In conclusion, we advocate for immediate surgical intervention when clinical suspicion of LAEF after RFA is high to prevent significant mortality. A teambased approach including interventional cardiology, radiology, anesthesiology, cardiac perfusionists, and cardiothoracic surgeons is invaluable and will hopefully lead to superior outcomes and avoid major catastrophic complications.

\section{References}

1 Sonmez B, Demirsoy E, Yagan N, et al. A fatal complication due to radiofrequency ablation for atrial fibrillation: atrio-esophageal fistula. Ann Thorac Surg 2003;76(1):281-283

2 Dagres N, Anastasiou-Nana M. Prevention of atrial-esophageal fistula after catheter ablation of atrial fibrillation. Curr Opin Cardiol 2011;26(1):1-5

3 Stöllberger C, Pulgram T, Finsterer J. Neurological consequences of atrioesophageal fistula after radiofrequency ablation in atrial fibrillation. Arch Neurol 2009;66(7):884-887

4 Siegel MO, Parenti DM, Simon GL. Atrial-esophageal fistula after atrial radiofrequency catheter ablation. Clin Infect Dis 2010;51(1): 73-76

5 Eitel C, Rolf S, Zachäus M, et al. Successful nonsurgical treatment of esophagopericardial fistulas after atrial fibrillation catheter ablation: a case series. Circ Arrhythm Electrophysiol 2013;6(4): 675-681 